\title{
Phase Transitions in One-Dimensional Nonequilibrium Systems
}

\author{
M. R. Evans \\ Department of Physics and Astronomy, \\ The University of Edinburgh, \\ Mayfield Road, \\ Edinburgh EH9 $3 J Z$, U.K.
}

Received 1 November 1999

\begin{abstract}
The phenomenon of phase transitions in one-dimensional systems is discussed. Equilibrium systems are reviewed and some properties of an energy function which may allow phase transitions and phase ordering in one dimension are identified. We then give an overview of the one-dimensional phase transitions which have been studied in nonequilibrium systems. A particularly simple model, the zero-range process, for which the steady state is known exactly as a product measure, is discussed in some detail. Generalisations of the model, for which a product measure still holds, are also discussed. We analyse in detail a condensation phase transition in the model and show how conditions under which it may occur may be related to the existence of an effective long-range energy function. It is also shown that even when the conditions for condensation are not fulfilled one can still observe very sharp crossover behaviour and apparent condensation in a finite system. Although the zero-range process is not well known within the physics community, several nonequilibrium models have been proposed that are examples of a zero-range process, or closely related to it, and we review these applications here.
\end{abstract}

\section{Introduction}

In recent years the study of nonequilibrium systems has come to the fore in statistical mechanics. Basically, one can consider two types of nonequilibrium systems: those relaxing towards thermal equilibrium and those held far from thermal equilibrium e.g. by the system being driven by some external field. In the present article we will be mainly concerned with the latter scenario.

To be more specific we define our nonequilibrium systems as those evolving through a local stochastic dynamics which a priori does not obey detailed balance, at least not with respect to any 'reasonable' energy function. The question of what is a reasonable energy function is a moot point. One might propose that the energy contains only local interactions, or is extensive, or is written down according to some physical principles; but any answer to the question is subjective. However, the basic point is that the nonequilibrium system is defined by its dynamics without regard to any concept of energy and it is the dynamics which should seem reasonable or 'physical'. This is distinct from an equilibrium system where the energy function should be 'physical' and the dynamics is usually defined in an ad hoc way simply to guarantee that one obtains the Gibbs-Boltzmann weight with the specified energy. The easiest way to do this is to use the detailed balance condition.
A natural way to construct a nonequilibrium steady state is to drive the system by forcing a current of some conserved quantity, for example energy or mass, through the system. Such systems are known as driven diffusive systems (DDS). The archetypal model was introduced Katz, Lebowitz and Spohn [1]. Basically it comprises a two dimensional Ising-like lattice gas evolving under conservative Kawasaki dynamics (spin exchange) and with a drive direction imposed. It has been shown that a continuous phase transition exists in the driven system, as is also the case in the undriven (Ising) system, but, most interestingly, one sees generic long range (power-law decay) correlations as opposed to the undriven systems where long-range correlations are only seen at criticality. Although exact results are not available for this system[2], it is often thought that generic power-law correlations are related to the existence of an effective long-range Hamiltonian for the system (see e.g. $[3,4]$ ).

More recently it has been realised that DDS in one dimension exhibit non-trivial behaviour. The interest has been from a fundamental viewpoint but also in the context of applications such as interface growth [5] and traffic flow modelling [6]. Also it turns out that problems of transport with a single-file constraint have long been of interest in biological contexts such as transport across membranes $[7,8]$ and the kinetics of biopolymerisation[9]. 
One intriguing feature of one-dimensional systems is the possibility of phase ordering and phase transitions. In recent years this possibility has begun to be explored and some examples are by now well studied. To appreciate the significance one should recall the general dictum that in one-dimensional equilibrium systems phase ordering and phase transitions do not occur (except in the limit of zero-temperature, or with long range interaction-see Section II). In the onedimensional nonequilibrium systems studied so far it appears that the presence of conserved quantities and an imposed drive are important in allowing ordering and phase transitions. However there still does not exist a general theoretical framework within which to understand the phenomena.

The purpose of this article is twofold. Firstly I wish to give a broad overview of phase transitions and phase ordering in one dimension-this is carried out in Section II. In particular, in Section II.1 we discuss the conditions under which ordering and phase transitions may occur in equilibrium systems i.e. the requisite properties of the energy function to allow such phenomena. Then in Section II.2 we catalogue some nonequilibrium, one dimensional systems which exhibit non-trivial phase behaviour

The second purpose is to discuss a very simple class of microscopic models, the zero-range processes [10,11], which are presented in Section III.1. For these models the steady state can be calculated exactly since it factorises into a product measure. There is some irony in the fact that the system has found widespread application in the modelling of nonequilibrium phenomena (see Section VI), although the zero-range process was originally introduced by Spitzer[10] as a dynamics which could lead to Gibbs measures. In Section IV we discuss generalisations of the basic model which also have a product measure steady state. We show in Section $\mathrm{V}$ how the model can exhibit a phase transition, that we shall refer to as a condensation transition, which is analysed in some detail. We also discuss an interesting sharp crossover phenomenon whereby models, although not fulfilling the conditions for strict condensation and phase ordering, may often appear to be in a condensed phase on a finite system. The simplicity of the system allows us to explore the roles of a conserved quantity, the presence of a drive and effective long-range energy functions. Conclusions are drawn in Section VII.

\section{Phase transitions in one dimension}

\section{II.1 One-dimensional equilibrium systems}

As mentioned above, it is received wisdom that in one-dimensional equilibrium systems phase transitions do not occur. In fact any careful statement of this requires a few caveats and, indeed, a general rigorous statement is hard to formulate (see [12] for a discussion).

Perhaps the best known argument is that of Landau and Lifshitz [13]. For simplicity, consider a one dimensional lattice of $L$ sites with two possible states, say $A$ and $B$, for each site variable. Let us assume the ordered phases, where all sites take state $A$ or all sites take state $B$, have the lowest energy, and assume a domain wall (a bond on the lattice which divides a region of $A$ phase from that of $B$ ) costs a finite amount of energy $\epsilon$. Then $n$ domain walls will cost energy $n \epsilon$ but the entropic contribution to the free energy due to the number or ways of placing $n$ walls on $L$ sites $\simeq n T[\ln (n / L)-1]$ for $1 \ll n \ll L$. Thus for any finite temperature a balance between energy and entropy ensures that the number of domain walls grows until it scales as $L$, that is, until the typical ordered domain size is finite.

Note that this argument relies on a finite energy cost for domain walls, and short range interactions so that one may ignore the interaction energy of domain walls. Indeed, the Ising model with long-range interactions decaying with distance as $J(r) \sim r^{-1-\sigma}$ has been well studied [14] and it has been demonstrated that the one-dimensional system orders at low temperatures for $\sigma \leq 1$ [15]. Also, of course we require non-zero temperature so that entropy comes into play, otherwise the two fully ordered states (ground states) would dominate the partition sum and the system would be frozen into them.

Another even simpler way of thinking of this is from a dynamical perspective. For a disordered state to order, domain walls must annihilate each other. However in one dimension no energy is gained by the two domain walls at opposite ends of a domain moving closer to one another; a domain always has two domain walls costing energy $2 \epsilon$ no matter what its size is. Therefore there is no effective force to eliminate domains and the system is disordered. Again, this argument requires a short range interaction so that one can ignore the energy of interaction of domain walls above some finite distance.

A more mathematical way of addressing the question of phase transition in $1 \mathrm{~d}$ is to use the transfer matrix technique [16]. For example, on a periodic onedimensional homogeneous system of $N$ sites, the partition sum can be written as the trace of a product of $N$ transfer matrices $T$ :

$$
Z=\operatorname{Trace}\left[T^{N}\right]=\sum_{\lambda} \lambda^{N}
$$

where $\lambda$ are the eigenvalues of the transfer matrix. Now, since the transfer matrix is finite and the entries are all positive the Perron-Frobenius theorem [17] tells us that the largest eigenvalue $\lambda_{\max }$ is non-degenerate. Thus, there can be no crossing of the largest eigenvalue as we vary some control parameter. Consequently the free energy $F \propto \lim _{N \rightarrow \infty}(\ln Z) / N=\lambda_{\max }$ is analytic and we have no phase transitions (which would be signalled by some non-analyticity of the free energy).

Again, there are exceptions to this reasoning i.e. when the Perron-Frobenius theorem no longer applies. 
This can occur when the transfer matrix becomes infinite due, for example, either to long range interactions or when the local degree of freedom at each lattice site is not restricted to a finite number of states e.g. [18]. (An extreme instance of the latter case is when we are actually considering a two dimensional system!) Another case when the Perron-Frobenius theorem does not apply is when the transfer matrix becomes reducible i.e. when there exist components of $T^{N}$ that are zero for all values of $N$. This can occur at zero temperature or when some interaction strengths are set to infinity, an example being the first order transition in the KDP model discussed in [19].

In this section we have discussed three arguments, presented here at different (low) levels of rigour, which all point to phase transitions in equilibrium onedimensional systems only being possible in the case of long-range interactions, zero-temperature limit or infinite interaction energies, or unbounded local variable at a site. As we shall see the situation for nonequilibrium systems is less restrictive although some parallels can be drawn.

\section{2 One-dimensional nonequilibrium systems}

Here we give an overview of one-dimensional systems where phase transitions and phase ordering may occur. We focus our attention on hopping particle models that, despite their simplicity, offer a wide range of non-trivial behaviour.

A simple one-dimensional model of a driven diffusive system is the asymmetric simple exclusion process (ASEP). Here particles hop in a preferred direction on a one-dimensional lattice with hard-core exclusion (at most one particle can be at any given site). Indicating the presence of a particle by a 1 and an empty site (hole) by 0 the dynamics comprises the following exchanges at nearest neighbour sites

$$
\begin{aligned}
& 10 \rightarrow 01 \text { with rate } 1 \\
& 01 \rightarrow 10 \text { with rate } q
\end{aligned}
$$

The open system was studied by Krug[20] and boundary induced phase transitions shown to be possible. Specifically one considers a lattice of $N$ sites where at the left boundary site (site 1) a particle is introduced with rate $\alpha$ if that site is empty, and at the right boundary site (site $N$ ) any particle present is removed with rate $\beta$. Thus the dynamical processes at the boundaries are

$$
\begin{aligned}
& \text { at site } 10 \rightarrow 1 \text { with rate } \alpha \\
& \text { at site } N 1 \rightarrow 0 \text { with rate } \beta \text {. }
\end{aligned}
$$

These boundary conditions force a steady state current of particles $J$ through the system. Phase transitions occur when $\lim _{N \rightarrow \infty} J$ exhibits non-analyticities. The steady state of this system was solved exactly for the totally asymmetric case $[21,22]$ and more recently for the general $q$ case $[23,24]$. When $q<1$ the phase diagram comprises three phases: a high-density phase where the current is limited by a low exit rate $\beta$ and takes the expression $J=\beta(1-q-\beta) /(1-q)$; a low-density phase where the current is limited by a low injection rate $\alpha$ and takes the expression $J=\alpha(1-q-\alpha) /(1-q)$; a maximal-current phase where both $\alpha, \beta>(1-q) / 2$ and the current is $J=(1-q) / 4$. In the maximal current phase generic long-range correlations exist, an example being the decay of particle density from the left boundary to the bulk value $1 / 2$ which is a power law $\sim 1 / x^{1 / 2}$ where $x$ is distance from the left boundary.

Clearly the presence of a conserved quantity and a drive, leading to non-zero current $J$ is crucial to the phase transition. Indeed, the qualitative phase diagram appears robust for stochastic one-dimensional driven systems [25]. For the case of no bulk drive $q=1[26,27]$, or 'reverse bias' $q>1$ [24] the current vanishes with increasing system size and there are no boundary-induced phase transitions.

The model has been generalised to two oppositely moving species of particle: one species is injected at the left, moves rightwards and exits at the right; the other species is injected at the right, moves leftwards and exits at the left [28]. Spontaneous symmetry breaking has been shown to occur, whereby for low exit rates $(\beta)$ the lattice is dominated by one of the species at any given time. In the low $\beta$ limit the mean flip time between the two symmetry-related states has been calculated analytically and shown to diverge exponentially with system size [29].

In these models the open boundaries can be thought of as inhomogeneities where the order parameter (particle density) is not conserved. Inhomogeneities which conserve the order parameter can be considered on a periodic system. Indeed a single defect bond on the lattice (through which particles hop more slowly) is sufficient to cause the system to separate into two macroscopic regions of different densities [30]: a high density region which can be thought of as a traffic jam behind the defect and a low density region in front of the defect. Here the presence of the drive appears necessary for the defect to induce the phase separation.

Moving defects (i.e. particles with dynamics different from that of the others) have also been considered and exact solutions obtained [31, 32, 33, 34, 35]. In the model studied in $[33,34,35]$, varying the rate at which the defect particle hops forward, denoted $\alpha$, and the rate at which it is overtaken and exchanges places with normal particles, denoted $\beta$, produces a phase diagram closely related to the open boundary problem. Moreover for low $\beta$ and high $\alpha$ there is a phase where the defect particle induces phase separation between a high density region behind it and a low density region in front of it.

For some of the examples discussed so far the steady state has been solved exactly by constructing a matrix product which is reviewed in [36]. This reveals that the steady state weights are very complicated functions of 
the particle number and positions. It does not appear easy to relate this to any concept of an energy function. Indeed, it has been shown that a matrix product state is non-Gibbsian [37].

A natural question to ask is whether systems related to the hopping particle models described so far, but without inhomogeneities, can exhibit phase ordering. A very simple model was introduced in [38] comprising three species of conserved particles, amongst which all possible exchanges are allowed. However a key feature is that the dynamics has a cyclic symmetry. To be specific let each site of a one-dimensional periodic lattice be occupied either by an $A, B$ or $C$ particle (there are no holes in this model). The dynamical exchanges are

$$
\begin{aligned}
& A B \rightarrow B A \text { with rate } q \\
& B A \rightarrow A B \text { with rate } 1 \\
& B C \rightarrow C B \text { with rate } q \\
& C B \rightarrow B C \text { with rate } 1 \\
& C A \rightarrow A C \text { with rate } q \\
& A C \rightarrow C A \text { with rate } 1
\end{aligned}
$$

and we will take $q<1$. For example, the hopping of an $A$ particle is biased to the right when it is an environment of $C$ s and it is biased to the left when it is in an environment of $B \mathrm{~s}$.

The phase separation observed in the model is rather easy to understand: if the system has separated into a domain of $A \mathrm{~s}$, followed by a domain of $B \mathrm{~s}$, followed by a domain of $C s$ (in that order), then the domain walls that are present $A B, B C, C A$ are all stable objects. This is clear from (4) since, for example, any $A$ particles which penetrate the $B$ domain will be driven backwards by the dynamics. On the other hand $B A$, $C B$ or $A C$ walls are all unstable objects and would be quickly eliminated by the dynamics.

In the special case of exactly equal numbers of $A, B$ and $C$ particles it was shown that the model actually obeys detailed balance with respect to a long range asymmetric, energy function. In fact the energy is nonextensive in the sense that most configurations have energies of order $N^{2}[38]$. The partition sum was calculated in the large $N$ limit (with $q$ fixed) and shown to depend linearly on $N$. This reflects the fact that the phase separation is into three pure domains and the partition sum is dominated by the $N$ equivalent translations of the structure comprising three pure domains. When the numbers of particles are not identical, detailed balance does not hold but the phase separation into pure domains remains. Similar behaviour has been found in other related models with conserving dynamics $[39,40]$. Another interesting model is where phase separation occurs on a quasi-one-dimensional system $(2 \times N$ sites) but not on a strictly one-dimensional system [41]. It should also be mentioned that systems with a cyclic symmetry but with non-conserving dynamics have been studied and shown to order into a frozen state [42].

Any discussion of nonequilibrium phase transitions is not complete without mentioning the most well known class, that of directed percolation. Various models are reviewed elsewhere in this volume[43] so here I just sketch the basic behaviour by referring to a particular model, the contact process $[44,45]$. Each site of a lattice is either empty or contains a particle. Particles are annihilated with rate 1 and particles are created at empty sites with rate $n \lambda / 2$ where $n$ is the number of occupied nearest neighbours of the site $(n=0,1,2)$. Note that the 'inactive state' where all sites are empty is an absorbing state. Above a critical value of $\lambda$ there is a finite probability that starting from a single particle on an infinite lattice, the system will remain active as $t \rightarrow \infty$. This phase transition has well-studied associated critical exponents and scaling behaviour. Moreover it appears to be a universality class in the sense that the same exponents are found in all systems, with the same symmetry and conservation laws, exhibiting a phase transition from an absorbing inactive state to an active state[45].

However as described so far the contact process is distinct from the other hopping particle models discussed in that on any finite lattice the absorbing state is reached in a finite time and is therefore the steady state. The active state only becomes available as a steady state on an infinite system. We mention briefly that it is in fact possible to define hopping particle models, similar in spirit to the nonequilibrium models discussed in previous paragraphs, that exhibit phase transitions connected with directed percolation. These models can have non-conserved order parameter [46] or conserved order parameter [47]. Although there are no absorbing states in these models, they have the common feature of certain microscopic processes being forbidden.

A final class of transitions in one-dimensional hopping particle models is that involving spatial condensation, whereby a finite fraction of the particles condenses onto the same site. Examples include the appearance of a large aggregate in models of aggregation and fragmentation[48] and the emergence of a single flock in dynamical models of flocking [49, 50]. In Section $\mathrm{V}$ we shall examine a very simple example of a condensation transition which occurs in the zero-range process and see how it is related to a defect induced transition.

\section{The zero-range process}

The zero-range process was first introduced into the mathematical literature as an example of interacting Markov processes [10]. Since then the mathematical achievements have been to prove existence theorems, invariant measures and hydrodynamic limits [44, 51].

It is not widely appreciated that the zero-range process has many physical applications; moreover it has often appeared incognito in a wide range of different contexts. Examples include the repton model of polymer dynamics with periodic boundary conditions [52]; a model of sandpile dynamics [53]; the backgammon 
model [54] for glassy dynamics due to entropic barriers; the drop-push model for the dynamics of a fluid moving through backbends in a porous medium [55]; microscopic models of step flow growth $[56,57]$ and a bosonic lattice gas [58]. We shall discuss some of these in the sequel. The zero-range process is also closely related to the more widely known asymmetric exclusion process $[10,44]$ as we shall describe below.

\section{III.1 Model definition}

In general one can consider the zero-range process on a lattice of arbitrary dimension, and of a (countably) infinite or finite number of sites. Initially Spitzer [10] considered a finite number of sites. However, subsequently most mathematical works tackle the invariant measure on an infinite system [11]. For our purposes, it is most convenient to consider a finite system, compute the steady state and only then take the limit of an infinite system. Note that the steady state $t \rightarrow \infty$ and the infinite volume limit do not necessarily commute e.g. on an infinite system the invariant measure (steady state) is not necessarily unique.

We consider a one-dimensional finite lattice of $M$ sites with sites labelled $\mu=1 \ldots M$ and periodic boundary conditions. Each site can hold an integer number of indistinguishable particles. The configuration of the system is specified by the occupation numbers $n_{\mu}$ of each site $\mu$. The total number of particles is denoted by $L$ and is conserved under the dynamics. The dynamics of the system is given by the rates at which a particle leaves a site $\mu$ (one can think of it as the topmost particle-see Fig. 1a). As our first example we assume it moves to the left nearest neighbour site $\mu-1$. The hopping rates $u(n)$ are a function of $n$ the number of particles at the site of departure. Some particular cases are: if $u(n)=n$ then the dynamics of each particle is independent of the others; if $u(n)=$ const for $n>0$ then the rate at which a particle leaves a site is unaffected by the number of particles at the site (as long as it is greater than zero). It is helpful to think of performing a Monte-Carlo simulation: in the $u(n)=n$ case at each update a particle would be picked at random and moved to its nearest neighbour site; in the $u(n)=$ constant case a site would be picked at random and a single particle moved to the nearest neighbour site.

A possible source of confusion in the definition of the model is that in [10] and some other papers the hop rates $u(n)$ are defined as the hop rate per particle at a site; thus $u(n)$ in those works are $1 / n$ of the $u(n)$ defined here.

The important attribute of the zero-range process is that it yields a steady state described by a product measure. By this it is meant that the steady state probability $P\left(\left\{n_{\mu}\right\}\right)$ of finding the system in configuration $\left\{n_{1}, n_{2} \ldots n_{M}\right\}$ is given by a product of factors $f\left(n_{\mu}\right)$ often referred to as marginals

$$
P\left(\left\{n_{\mu}\right\}\right)=\frac{1}{Z(M, L)} \prod_{\mu=1}^{M} f\left(n_{\mu}\right)
$$

Here the normalisation $Z(M, L)$ is introduced so that the sum of the probabilities for all configurations, with the correct number of particles $L$, is one. We shall explore later in Section $\mathrm{V}$ the interesting possibilities afforded by the form (5).

In the basic model described above, $f(n)$ is given by

$$
\begin{aligned}
f(n) & =\prod_{m=1}^{n} \frac{1}{u(m)} \text { for } n \geq 1 \\
& =1 \text { for } n=0
\end{aligned}
$$

Note that $f(n)$ is defined only up to a multiplicative constant and we could have included a factor $z^{n}$ in (6). Later this factor reappears as a fugacity in Section V.

The proof of $(5,6)$ is, happily, straightforward. One simply considers the stationarity condition on the probability of a configuration (probability current out of the configuration due to hops is equal to probability current into the configuration due to hops):

$$
\sum_{\mu} \theta\left(n_{\mu}\right) u\left(n_{\mu}\right) P\left(n_{1} \ldots n_{\mu} \ldots n_{L}\right)=\sum_{\mu} \theta\left(n_{\mu}\right) u\left(n_{\mu+1}+1\right) P\left(n_{1} \ldots n_{\mu}-1, n_{\mu+1}+1 \ldots n_{L}\right) .
$$

We have included the Heaviside function to highlight that it is the sites with $n>1$ that allow exit from the configuration (lhs of (7)) but also allow entry to the configuration (rhs of ( 7 )). Equating the terms $\mu$ with $\mu>1$ and cancelling common factors assuming (5), results (for $n_{\mu} \geq 1$ ) in

$$
u\left(n_{\mu}\right) f\left(n_{\mu-1}\right) f\left(n_{\mu}\right)=u\left(n_{\mu+1}+1\right) f\left(n_{\mu}-1\right) f\left(n_{\mu+1}+1\right)
$$

This equality can be recast as

$$
u\left(n_{\mu}\right) \frac{f\left(n_{\mu}\right)}{f\left(n_{\mu}-1\right)}=u\left(n_{\mu+1}+1\right) \frac{f\left(n_{\mu+1}+1\right)}{f\left(n_{\mu+1}\right)}=\mathrm{constant}
$$


Setting the constant equal to unity implies

$$
f\left(n_{\mu}\right)=\frac{f\left(n_{\mu}-1\right)}{u\left(n_{\mu}\right)}
$$

and iterating (10) leads to (6) where we have chosen $f(0)=1$.

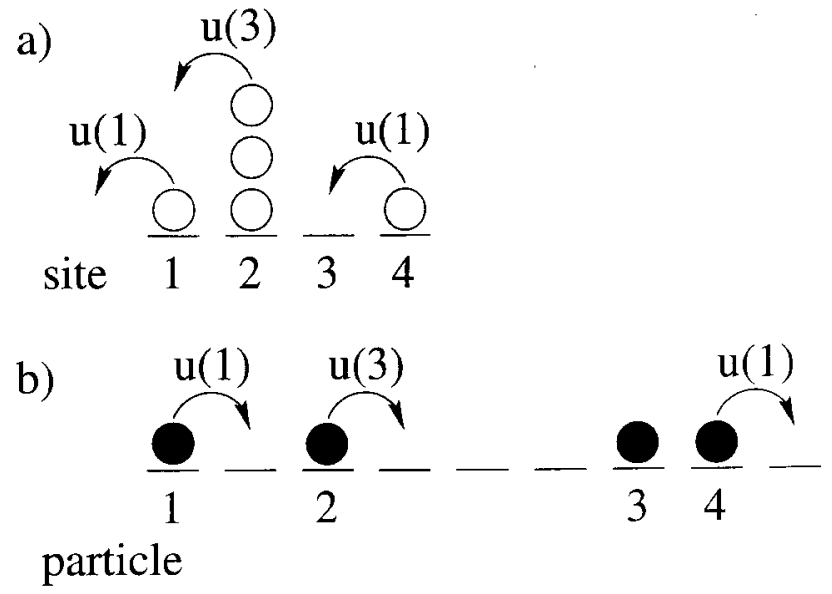

Figure 1. Equivalence of zero range process and asymmetric exclusion process.

\section{III.2 Relation to the asymmetric exclusion process}

There exists an exact mapping from a zero-range process to an asymmetric exclusion process. This is illustrated in Fig. 1. The idea is to consider the particles of the zero-range process as the holes (empty sites) of the exclusion process. Then the sites of the zero-range process become the moving particles of the exclusion process. This is possible because of the preservation of the order of particles under the simple exclusion dynamics. Note that in the exclusion process we have $M$ particles hopping on a lattice of $M+L$ sites

An interesting feature of the mapping is that it converts a model where the local degree of freedom can take unbounded values (particle number in the zerorange process) to a model where the local site variable is restricted to two values. On the other hand, a hopping rate $u(m)$ which is dependent on $m$ corresponds to a hopping rate in the exclusion process which depends on the gap to the particle in front. So in principle the particles can feel each other's presence and it is possible to have a long-range interaction.

\section{Generalizations}

We now show how the totally asymmetric, homogeneous zero-range process we have considered so far may be generalised yet retain steady states of a similar form to $(5,6)$.

\section{IV.1 Inhomogeneous system}

First we consider an inhomogeneous system by which we mean the hopping rates are site dependent: the hopping rate out of site $\mu$ when it contains $n_{\mu}$ particles is $u_{\mu}\left(n_{\mu}\right)$. It is easy to check that the steady state is simply modified to

$$
P\left(\left\{n_{\mu}\right\}\right)=\frac{1}{Z(M, L)} \prod_{\mu=1}^{L} f_{\mu}\left(n_{\mu}\right)
$$

where $f_{\mu}$ are given by

$$
\begin{aligned}
f_{\mu}(n) & =\prod_{m=1}^{n} \frac{1}{u_{\mu}(m)} \quad \text { for } \quad n \geq 1 \\
& =1 \quad \text { for } \quad n=0
\end{aligned}
$$

The proof is identical to that given above for the homogeneous case, with the trivial replacement of $u\left(n_{\mu}\right)$ by $u_{\mu}\left(n_{\mu}\right)$

\section{IV.2 Discrete Time Dynamics}

A further generalisation is to the case of discrete time dynamics. This has been studied in [59] in the context of a disordered asymmetric exclusion process. Here we translate the results into the zero-range process. Rather than processes occurring with a rate, time is counted in discrete steps and at each time step events occur with certain probabilities.

In the case of Parallel Dynamics, at each time-step all sites are updated. One particle from each site $\mu$ is moved to the left, each with probability $p_{\mu}\left(n_{\mu}\right)$ where $n_{\mu}$ is the number of particles at the site before the update. Note that the particles move simultaneously and particles do not move more than one site.

It turns out that the steady state again has the form (11). It was shown in [59] that

$$
\begin{aligned}
f_{\mu}(n) & =1-p_{\mu}(1) \text { for } n=0 \\
& =\frac{1-p_{\mu}(1)}{1-p_{\mu}(n)} \prod_{m=1}^{n} \frac{1-p_{\mu}(m)}{p_{\mu}(m)} \text { for } n>0 .
\end{aligned}
$$

To recover the continuous time dynamics we can call the interval between time steps $d t$ and let $p_{\mu}\left(n_{\mu}\right)=$ $u_{\mu}\left(n_{\mu}\right) d t$. Then continuous time dynamics is given by the limit $d t \rightarrow 0$ and, to within a constant factor $d t^{n}$, (13) recovers (12). In this way one can interpolate between discrete time, parallel dynamics and continuous time dynamics.

In [59] ordered sequential updating schemes were also considered. These are discrete time updating schemes were one site is updated at a time, but the sites are updated in a fixed order. The steady states for the 
forwards and backwards updating sequences were derived and it turns out they too have the form (11) with $f_{\mu}$ taking an expression related to the parallel case (13).

\section{IV.3 Arbitrary Network}

In the original paper paper of Spitzer[10] some more general versions of the zero range process were considered. Here we discuss one interesting case which serves to generalise the (totally asymmetric) zero range process defined above to a process on a more general lattice or for any finite collection of points with a prescribed transition matrix for the dynamics of a single particle [11].

In this case the rate of hopping of a particle at site $\mu$ containing $n_{\mu}$ particles is equal to $u_{\mu}\left(n_{\mu}\right)$ and the probability that a particle leaving site $\mu$ will move to site $\nu$ is denoted $W(\mu \rightarrow \nu)$. Thus the probability that in time $d t$ a particle at $\mu$ moves to $\nu$ is

$$
u_{\mu}\left(n_{\mu}\right) W(\mu \rightarrow \nu) d t
$$

Note that the probabilities $W(\mu \rightarrow \nu)$ define a stochastic matrix for a single particle moving on a finite collection of $M$ sites and we take

$$
\sum_{\nu} W(\mu-\nu)=1
$$

so that probability is conserved. We refer to the collection of points together with the prescribed transition matrix $W(\mu \rightarrow \nu)$ as the network.

We assume that the transition matrix is irreducible (i.e. the particle can pass from any given point to any other after sufficient time and the system is ergodic) so that we have a unique steady state probability for the single particle problem:

$$
s_{\nu}=\sum_{\mu} s_{\mu} W(\mu \rightarrow \nu)
$$

We now show that the steady state for the manyparticle problem defined above is given by (11) where now $f_{\mu}(n)$ is given by

$$
\begin{aligned}
f_{\mu}(n) & =\prod_{m=1}^{n}\left[\frac{s_{\mu}}{u_{\mu}(m)}\right] \quad \text { for } n \geq 1 \\
& =1 \quad \text { for } n=0
\end{aligned}
$$

The proof is again a straightforward generalisation of that of Section III. Equation (7) is modified to

$$
\sum_{\mu} \theta\left(n_{\mu}\right) u_{\mu}\left(n_{\mu}\right) P\left(n_{1} \ldots n_{L}\right)=\sum_{\mu} \sum_{\nu \neq \mu} \theta\left(n_{\mu}\right) p(\nu \rightarrow \mu) u_{\nu}\left(n_{\nu}+1\right) P\left(n_{1} \ldots n_{\nu}+1 \ldots n_{\mu}-1 \ldots n_{L}\right) .
$$

Equating the terms $\mu$ on each side of (18), assuming (11) and cancelling common factors yields

$$
\theta\left(n_{\mu}\right) u_{\mu}\left(n_{\mu}\right) f_{\mu}\left(n_{\mu}\right)=\theta\left(n_{\mu}\right) \sum_{\nu \neq \mu} W(\nu \rightarrow \mu) u_{\nu}\left(n_{\nu}+1\right) f_{\mu}\left(n_{\mu}-1\right) \frac{f_{\nu}\left(n_{\nu}+1\right)}{f_{\nu}\left(n_{\nu}\right)}
$$

Inserting (17) leads to the condition

$$
s_{\mu}=\sum_{\nu \neq \mu} s_{\nu} W(\nu \rightarrow \mu)
$$

which is the same as the single particle steady state condition (16).

A simple case considered by Spitzer is when $W(\mu \rightarrow \nu)$ is a doubly stochastic matrix which is defined by the property

$$
\sum_{\nu} W(\nu \rightarrow \mu)=\sum_{\mu} W(\nu \rightarrow \mu)=1 .
$$

Equations (21) and (16) then imply that the single particle problem has a homogeneous steady state $s_{\mu}=$ constant.

Let us also discuss an example where the single particle problem has an inhomogeneous steady state. We consider a one-dimensional lattice where hops to the left and right neighbours are allowed but with probabilities that depend on the site. Thus, we may write

$$
\begin{aligned}
W(\mu \rightarrow \nu) & =q_{\mu} \text { for } \nu=\mu+1 \\
& =1-q_{\mu} \text { for } \nu=\mu-1 \\
& =0 \text { otherwise. }
\end{aligned}
$$

The steady state of the single particle problem (random walker on a disordered one dimensional lattice [60])

$$
s_{\mu}=\left(1-q_{\mu+1}\right) s_{\mu+1}+q_{\mu-1} s_{\mu-1}
$$

can be solved and one obtains

$$
s_{\mu}=\left[\sum_{i=0}^{M-1} \frac{1}{q_{\mu-i}} \prod_{\nu=0}^{i} \frac{q_{\mu-\nu}}{1-q_{\mu-\nu}}\right] .
$$

This network is relevant to the disordered onedimensional exclusion process studied in [61, 62, 63]. 
The sites in the present model correspond to the particles in the exclusion process which each have their own forward and backward hopping rates. Another, particular instance of this network occurs in [52], where a repton model of gel electrophoresis is studied in the case of periodic boundary conditions (see Section VI).

In special cases of the zero-range process detailed balance may hold. The condition for this is

$$
u_{\mu}\left(n_{\mu}\right) W(\mu \rightarrow \nu) P\left(n_{1} \ldots n_{L}\right)=u_{\nu}\left(n_{\nu}+1\right) W(\nu \rightarrow \mu) P\left(n_{1} \ldots n_{\nu}+1 \ldots n_{\mu}-1 \ldots n_{L}\right) .
$$

Substituting the form $(11,17)$ leads to the condition

$$
s_{\mu} W(\mu \longrightarrow \nu)=s_{\nu} W(\nu \longrightarrow \mu)
$$

which is just the detailed balance condition for the single particle problem.

An interesting consequence of the form of the steady state (17) is that it allows one to relate an arbitrary zero-range process to a model obeying detailed balance. The idea is that if detailed balance doesn't hold, we can always define a new zero-range process (to be denoted by a prime) with the same steady state, but with a different dynamics obeying detailed balance. To do this, we solve the single particle problem (16) for the original model to obtain $s_{\mu}$. For any collection of points we can always define a new single particle transition matrix $W^{\prime}(\mu \rightarrow \nu)$ that satisfies detailed balance with respect to a homogeneous steady state $\left(s_{\mu}^{\prime}=\right.$ constant $)$. The new model is defined by a new set of hopping rates $u_{\mu}^{\prime}(m)=u_{\mu}(m) s_{\mu}^{\prime} / s_{\mu}$ together with the new transition matrix $W^{\prime}(\mu \rightarrow \nu)$. It is easy to check from (17) that the new model has the same steady state as the original.

Thus, within the realm of zero-range processes, to the steady state of any nonequilibrium model we can always identify a model satisfying detailed balance and therefore an energy function. Of course, although the steady states are the same, there is no reason for the dynamical behaviour of the two systems to be related. To clarify this point we will discuss a simple example in Section .

The marginals (17) have the interesting structure of being a product of a term $\left(s_{\mu}\right)^{n}$ that depends on the nature of the network and a term involving the product of $u_{\nu}(m)$ which reflects the interactions at the site. The network can represent an arbitrary dimensional lattice or the effects of disorder, the only difficulty to surmount in obtaining the steady state is the solution of the single particle problem.

\section{Condensation Transitions}

We now proceed to analyse the steady states of form (11) and the condensation transition that may occur. The important quantity to consider is the normalisation $Z(M, L)$ as it plays the role of the partition sum. The normalisation is defined through the condition

$$
Z(M, L)=\sum_{n_{1}, n_{2} \ldots n_{M}} \delta\left(\sum_{\mu} n_{\mu}-L\right) \prod_{\mu=1}^{M} f_{\mu}\left(n_{\mu}\right)
$$

where the $\delta$ function enforces the constraint of $L$ particles. The normalisation may be considered as the analogue of a canonical partition function of a thermodynamic system.

We define the 'speed' $v$ as the average hopping rate out of a site

$$
\begin{aligned}
v & =\frac{1}{Z(M, L)} \sum_{n_{1}, n_{2} \ldots n_{M}} \delta\left(\sum_{\mu} n_{\mu}-L\right) u\left(n_{1}\right) \prod_{\mu=1}^{M} f_{\mu}\left(n_{\mu}\right) \\
& =\frac{Z(M, L-1)}{Z(M, L)}
\end{aligned}
$$

where we have used $(11,12)$. Note that $(30)$ tells us that the speed is independent of site and thus may be considered a conserved quantity in the steady state of the system. In the totally asymmetric system considered in Section III.1 the speed is equal to the current of particles flowing between neighbouring sites and is clearly a conserved quantity in the steady state. More generally, however, the speed is not equal to the current and the fact that the speed is a conserved quantity is not a priori obvious. For example, in a system obey- 
ing detailed balance the (net) current is zero, but the speed as defined above remains finite. The speed is a ratio of partition functions of different system sizes (30) and corresponds to a fugacity, as we shall see below.

We will consider also the probability distribution of the number of particles at a site, taken here to be site 1

$$
\begin{aligned}
P_{1}\left(n_{1}=x\right) & =\frac{1}{Z(M, L)} \sum_{n_{2} \ldots n_{M}} \delta\left(x+n_{2}+\ldots n_{M}-L\right) f_{1}(x) \prod_{\mu=2}^{M} f_{\mu}\left(n_{\mu}\right) \\
& =f_{1}(x) \frac{Z(M-1, L-x)}{Z(M, L)}
\end{aligned}
$$

(where $Z(M-1, L-x)$ is the partition function for a system with site 1 removed). In general the probability distribution is site dependent but for a homogeneous system $\left(f_{\mu}\right.$ independent of $\left.\mu\right)$ it will be the same for all sites.

We now use the integral representation of the delta function to write the partition function as

$$
Z(M, L)=\oint \frac{d z}{2 \pi i} z^{-(L+1)} \prod_{\mu=1}^{M} F_{\mu}(z),
$$

where

$$
F_{\mu}(z)=\sum_{m=0}^{\infty} z^{m} f_{\mu}(m) .
$$

For large $M, L(32)$ is dominated by the saddle point of the integral and the value of $z$ at the saddle point is the fugacity. The equation for the saddle point reduces to

$$
\frac{L}{M}=\frac{z}{M} \sum_{\mu=1}^{M} \frac{\partial}{\partial z} \ln F_{\mu}(z)
$$

which, defining $\phi=L / M$, can be written as

$$
\phi=\frac{z}{M} \sum_{\mu=1}^{M} \frac{F_{\mu}^{\prime}(z)}{F_{\mu}(z)} .
$$

In the thermodynamic limit,

$$
M \rightarrow \infty \text { with } L=\phi M,
$$

where the density $\phi$ is held fixed, the question is whether a valid saddle point value of $\mathrm{z}$ can be found from (35). We expect that for low $\phi$ the saddle point is valid but, as we shall discuss, there exists a maximum value of $z$ and if at this maximum value the rhs of (35) is finite, then for large $\phi(35)$ cannot be satisfied. We now consider separately, and in more detail, how condensation may occur in the inhomogeneous and the homogeneous case.

\section{V.1 Inhomogeneous case}

In general, the inhomogeneous case i.e. where $F_{\mu}(z)$ depends on the site $\mu$ through (17), is difficult to analyse. Here we would just like to give an idea of how a condensation transition may occur by discussing a simple example. We then go on to analyse perhaps the simplest example of a condensation transition: a single inhomogeneous site [62].

First we take the general model discussed in Section IV.3 and set $u_{\mu}(m)=u_{\mu}$ for $m>0$ i.e. the hopping rate does not depend on the number of particles at a site. We consider doubly stochastic transition matrices $W(\mu \rightarrow \nu)$ (see Eq. 21) so that we may take $s(\mu)=$ constant and without loss of generality we set the constant equal to one. For the moment we do not specify further the transition matrix; later we will discuss two specific examples one obeying detailed balance and one not. Under these conditions $f_{\mu}$ is given by

$$
f_{\mu}(n)=\left(\frac{1}{u_{\mu}}\right)^{n_{\mu}}
$$

and the probability of occupancies $\left\{n_{1}, n_{2}, \ldots, n_{M}\right\}$ is

$$
P\left(\left\{n_{1}, n_{2}, \ldots, n_{M}\right\}\right)=\frac{1}{Z(M, L)} \prod_{\mu=1}^{M}\left(\frac{1}{u_{\mu}}\right)^{n_{\mu}}
$$

The mapping to an ideal Bose gas is evident: the $L$ particles of the zero-range process are viewed as Bosons which may reside in $M$ states with energies $E_{\mu}$ determined by the site hopping rates: $\exp \left(-\beta E_{\mu}\right)=1 / u_{\mu}$. Thus the ground state corresponds to the site with the lowest hopping rate. The normalisation $Z(M, L)$ is equivalent to the canonical partition function of the 
Bose gas. We can sum the geometric series (33) to obtain $F_{\mu}$ and $F_{\mu}^{\prime}$ then taking the large $M$ limit allows the sum over $\mu$ to be written as an integral

$$
\phi=\int_{u_{\min }}^{\infty} d u \mathcal{P}(u) \frac{z}{u-z}
$$

where $\mathcal{P}(u)$ is the probability distribution of site hopping rates with $u_{\text {min }}$ the lowest possible site hopping rate. Interpreting $\mathcal{P}(u)$ as a density of states, equation (39) corresponds to the condition that in the grand canonical ensemble of an ideal Bose gas the number Bosons per state is $\phi$. The theory of Bose condensation [64] tells us that when certain conditions on the density of low energy states pertain we can have a condensation transition. Then (35) can no longer be satisfied and we have a condensation of particles into the ground state, which is here the site with the slowest hopping rate. This case is discussed further, in the context of an asymmetric exclusion process on an infinite system, by J. Krug in this volume [65].
We now turn to the simplest case of an inhomogeneous system: site 1 has $u_{1}=p$ while the other $M-1$ particles have hopping rates $u_{\mu}=1$ when $\mu>1$. It is easy to see that (11) simplifies to

$$
P\left(\left\{n_{\mu}\right\}\right)=\frac{1}{Z(M, L)} \frac{1}{p^{n_{1}}}
$$

In this case the normalisation $Z(M, L)$ is easy to calculate combinatorially:

$$
\begin{aligned}
Z(M, L) & =\sum_{n_{1}, n_{2} \ldots n_{M}} \delta\left(\sum_{\mu} n_{\mu}-L\right) p^{-n_{1}} \\
& =\sum_{n_{1}=0}^{L}\left(\begin{array}{c}
L+M-n_{1}-2 \\
M-2
\end{array}\right) p^{-n_{1}}
\end{aligned}
$$

yielding an exact expression for the speed through (30). In the thermodynamic limit the sum (41) is dominated by $n_{1} \sim \mathcal{O}(1)$ for $\phi<p /(1-p)$ and $n_{1} \sim \mathcal{O}(L)$ for $\phi>p /(1-p)$ and it can be shown that

$$
\begin{array}{lrl}
\text { for } \phi<\frac{p}{1-p} & Z(M, L) \simeq\left(\begin{array}{c}
L+M \\
M
\end{array}\right) \frac{1}{1+\phi} \frac{p}{p-\phi(1-p)} & \text { and } v \rightarrow 1-\rho \\
\text { for } \phi>\frac{p}{1-p} & Z(M, L) \simeq p^{-L}(1-p)^{-(M-1)} & \text { and } v \rightarrow \phi /(1+\phi)
\end{array}
$$

In the high density phase, defined by (43) we have a condensate since the average number of particles at site 1 is $\left\langle n_{1}\right\rangle / L=\phi-p /(1-p)$. In the low density phase (42) the particles are evenly spread between all sites and we will refer to it as the homogeneous phase.

We now discuss two models which both have this steady state: a driven system and a system obeying detailed balance. This provides an illustration of the idea discussed in Section IV.3 whereby a zero range process not obeying detailed can be related to one obeying detailed balance.

First we take the totally asymmetric model so that particles move to the site to the left: the transition matrix is

$$
W(\mu \rightarrow \nu)=\delta_{\nu, \mu-1}
$$

So this model is similar to that discussed in Section III.1, and a mapping to a totally asymmetric exclusion process can be made in the same way as Section III.2. The equivalent exclusion process is illustrated in Fig. 2. We see that the equivalent exclusion process is system of hard-core particles hopping to the right, one particle being slower than the rest. The interpretation of the two phases within the context of the exclusion process is that in the condensed phase (for the exclusion process a low density of particles) a 'traffic jam' forms behind the slow particle and the slow particle has a finite fraction of the lattice as 'empty road' ahead. Whereas in the homogeneous phase (a high density of particle for the exclusion process) the particles are roughly evenly spaced.

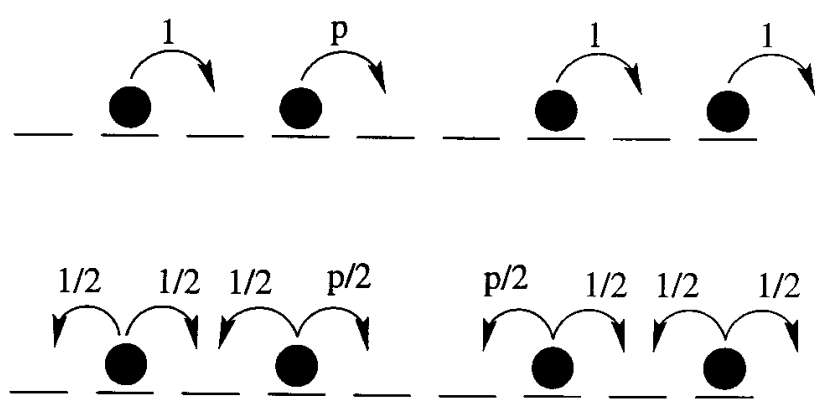

Figure 2. A totally asymmetric exclusion model (upper) and a model with zero current (lower) that have equivalent steady states leading to (41).

On the other hand one may consider the case where the one particle problem is a symmetric random walk so that the system obeys detailed balance. The transition matrix is given by

$$
W(\mu \rightarrow \nu)=\frac{1}{2} \delta_{\nu, \mu-1}+\frac{1}{2} \delta_{\nu, \mu+1} .
$$


When we map this system to a simple exclusion process we see from Fig. 2 that we have a system of particles, the bulk of which perform a symmetric exclusion dynamics but with two adjacent asymmetric particles: the left one biased to the left and the right one biased to the right. In the condensed phase the gap between these particles diverges. Previously a single asymmetric particle in a sea of symmetric particles has been studied [66] but in that case there is no phase transition. At first it seems that we have found a counterexample to the received wisdom that no phase transition should occur in an equilibrium system, since we have a condensation transition in a model with local dynamics obeying detailed balance. Inferring an energy function from the steady state (40) through the following equation

$$
\exp [-(\beta E)]=\exp \left[-\left(x_{2}-x_{1}\right) \ln p\right]
$$

reveals that our effective energy increases linearly with distance $x_{2}-x_{1}$ between the two asymmetric particles. Therefore the energy is 'unphysical' in that it has very long range interactions. Thus the phase transition can be rationalised within the categories of exceptions discussed in Section II.1.

We have seen that this simplest example of a condensation transition (a single inhomogeneous site in the zero range process) is exhibited both in a driven model and also in a model obeying detailed balance but with long-range energy function. Again it should be stressed that although the steady states of these two models are equivalent, the dynamical properties should be very different. For example in the homogeneous phase of the driven model we expect asymmetric exclusion like behaviour and the dynamic exponent should be $3 / 2$ implying relaxation times of $M^{3 / 2}$ on a finite system. However in the homogeneous phase of the model obeying detailed balance we expect symmetric exclusion like behaviour and the dynamic exponent to be 2 implying relaxation times of $M^{2}[67]$.

\section{V.2 Homogeneous case}

We now consider the homogeneous zero-range process where in (14) the hopping rates are site independent and the single particle problem (16) has a homogeneous steady state $s_{\mu}=1$ [68]. A similar analysis has been carried out in the context of balls-in-boxes and branched polymer models [69].

In the present case, (33) is independent of $\mu$ and reads

$$
F(z)=\sum_{n=0}^{\infty} \prod_{m=1}^{n}\left[\frac{z}{u(m)}\right]
$$

The fugacity $z$ must be chosen so that $F$ converges or else we could not have performed (33). Therefore $z$ is restricted to $z \leq \beta$ where we define $\beta$ to be the radius of convergence of $F(z)$. From (44) we see that $\beta$ is the limiting value value of the $u(m)$ i.e. the limiting value of the hopping rate out of a site for a large number of particles at a site. We interpret (35) as giving a relation between the density of holes (number of holes per site) and the fugacity $z$. The saddle point condition (35) becomes

$$
\phi=\frac{z F^{\prime}(z)}{F(z)}
$$

Given that the rhs of (45) is a monotonically increasing function of $z$ (which is not difficult to prove) we deduce that density of particle increases with fugacity. However if at $z=\beta$, the maximum allowed value of $z$, the rhs of (45) is still finite then one can no longer solve for the density and one must have a condensation transition. Physically, the condensation would correspond to a spontaneous symmetry breaking where one of the sites is spontaneously selected to hold a finite fraction of the particles.

Thus, for condensation to occur (i.e. when $\phi$ is large enough for (45) not to have a solution for the allowed values of $z$ ) we require

$$
\lim _{z \rightarrow \beta} \frac{F^{\prime}(z)}{F(z)}<\infty .
$$

We now assume that $u(n)$ decreases uniformly to $\beta$ in the large $n$ limit as

$$
u(n)=\beta(1+\zeta(n))
$$

where $\zeta(n)$ is a monotonically decreasing function. Analysis of the series

$$
\begin{aligned}
& F(\beta)=\sum_{n=0}^{\infty} \exp \left\{-\sum_{m=1}^{n} \ln [1+\zeta(m)]\right\} \\
& F^{\prime}(\beta)=\sum_{n=0}^{\infty} n \exp \left\{-\sum_{m=1}^{n} \ln [1+\zeta(m)]\right\}
\end{aligned}
$$

reveals that the condition for condensation is simply that $F^{\prime}(\beta)$ is finite and this occurs if $u(n)$ decays to $\beta$ more slowly than $\beta(1+2 / n)$. (This is easiest to see by expanding $\ln [1+\zeta]$ and approximating the sum over $m$ by an integral in (48).)

In order to fit this result into the picture of Section Section II.1 one can argue that since the condensate has an extensive number of particles at a site, the local site variable is unbounded. Therefore the "no phase transition rule' does not apply. One also gains insight by translating the results into the language of the simple exclusion process. In this context we can have condensation if the hop rate of a particle into a gap of size $n$ decays as $\beta(1+2 / n)$ therefore there is an effective long range interaction.

\section{V.3 Sharp crossover phenomena}

Having discussed the case where a true phase transition occurs we now consider a homogeneous example 
where, although there is no strict condensation transition, some interesting crossover phenomena occur [68].

Consider

$$
\begin{aligned}
& u(n)=1 \text { for } n<r \\
& u(n)=\beta \text { for } n \geq r .
\end{aligned}
$$

One can interpret these hop rates as meaning that a site only distinguishes whether it contains greater than $r$ particles. When we use the mapping of Section III.2 to a totally asymmetric exclusion process $r$ becomes the range of the interaction in the sense that it is the number of sites ahead up to which a particle in the exclusion process distinguishes.

When these hopping rates are inserted in (44) one obtains

$$
F(z)=\sum_{n=0}^{r-1} z^{n}+\sum_{n=r}^{\infty} z^{n} \beta^{r-n-1}
$$

Performing the geometric series readily yields

$$
\begin{aligned}
F(z) & =\frac{1-z^{r}}{1-z}+\frac{z^{r}}{\beta-z} \\
z F^{\prime}(z) & =z\left[\frac{1-z^{r}}{(1-z)^{2}}-\frac{r z^{r-1}}{1-z}+\frac{z^{r}}{(\beta-z)^{2}}+\frac{r z^{r-1}}{(\beta-z)}\right] .
\end{aligned}
$$

Then we find the condition (45) can be written after a little algebra as

$$
\begin{aligned}
(\beta-z)^{2}[\phi-z(1+\phi)]= & z^{r}(1-\beta)[(1+\beta-2 z) z-\phi(1-z)(\beta-z)] \\
& +r z^{r}(1-z)(\beta-z)(1-\beta)
\end{aligned}
$$

Therefore for large $r$ we find the solutions

$$
\begin{array}{ccc}
\text { for } \phi<\frac{\beta}{1-\beta} & z \simeq \frac{\phi}{1+\phi}-r\left(\frac{\phi}{1+\phi}\right)^{r} \frac{1-\beta}{(1+\phi)(\phi-\beta(1+\phi))} \\
\text { for } \phi & =\frac{\beta}{1-\beta} & z \simeq \beta-\beta^{(1+r) / 3}(1-\beta) \\
\text { for } \phi>\frac{\beta}{1-\beta} & \quad z \simeq \beta-\beta^{(1+r) / 2} \frac{1-\beta}{(\phi-\beta(1+\phi))^{1 / 2}}
\end{array}
$$

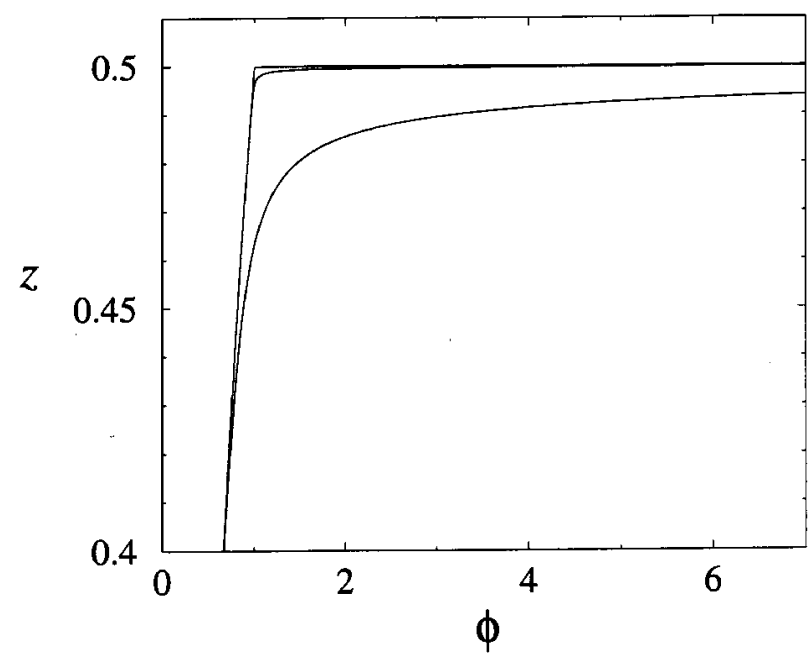

Figure 3. Solutions to (54) for $\beta=0.5$ and $r=10,20,30$ (increasing in sharpness of curve).

Thus we see as $r \rightarrow \infty$ we have two phases: a high density phase $\phi>\beta /(1-\beta)$ where the speed is $\beta$ and a low density phase where the speed is $\phi /(1+\phi)$. In fact these phases correspond exactly to those of the single defect problem discussed in the previous subsection $(42,43)$ with $\beta$ playing the role of $p$. For finite $r, z$ is actually a smoothly increasing function of $\phi$ but we see from $(55,57)$ that the curve sharpens as $r$ increases. This is illustrated in Fig. 3 where the numerical solution to (54) is plotted is plotted for $r=10,20,30$. One sees a dramatic sharpening as $r$ increases leading to a sharp crossover between a low density and high density regime.

In order to see the effects of this sharp crossover it is interesting to consider the particle number probability distribution (31) which for this system is site independent and given by

$$
\begin{aligned}
P(x) & \sim z^{x} \quad \text { for } \quad x<r \\
& \sim(z / \beta)^{x} \text { for } x \geq r .
\end{aligned}
$$

One can think of this as a sum of two distributions, one for poorly occupied sites $x<r$ and one for well occupied sites $x \geq r$. When $\phi>\beta /(1-\beta)$ the probability 
distribution for large $x>r$ goes as

$$
P(x) \sim \exp \left\{-x \beta^{(1+r) / 2} \frac{1-\beta}{(\phi-\beta(1+\phi))^{1 / 2}}\right\}
$$

so that the typical occupancy of well-occupied sites goes as $\beta^{-r / 2}$. Taking, for example $\beta=0.1$ and $r=10$ leads to a typical occupancy of $\sim 10^{5}$. Therefore to simulate the model one requires a number of particles very much larger than this! If care is not taken to do this, and the total number of particles in the system is comparable to the typical occupancy, one would have an apparent condensate on a finite system.

An example of this phenomenon was studied recently within the context of a 'bus route model' [70]. There the underlying motivation was to consider how a non-conserved quantity could mediate an effective longrange interaction amongst a conserved quantity in a driven system with a strictly local dynamical rule. The model considered was defined on a $1 d$ lattice. Each site (bus-stop) is either empty, contains a bus (a conserved particle) or contains a passenger (non-conserved quantity). The dynamical processes are that passengers arrive at an empty site with rate $\lambda$; a bus moves forward to the next stop with rate 1 if that stop is empty; if the next stop contains passengers the bus moves forward with rate $\beta$ and removes the passengers. Since the buses are conserved, there is a well defined steady state average speed $v$. This fact can be used to integrate out the non-conserved quantity (passengers) within a mean-field approximation. The idea is that a bus stop, next to bus 1 say, will last have been visited by a bus (bus 2) a mean time ago of $n / v$ where $n=x_{2}-x_{1}$ is the distance between bus 2 and bus 1 . Therefore the mean-field probability that the site next to bus 1 is not occupied by a passenger is $\exp (-\lambda n / v)$. From this probability an effective hopping rate for a bus into a gap of size $n$ is obtained by averaging the two possible hop rates $1, \beta$ :

$$
u(n)=\beta+(1-\beta) \exp (-\lambda n / v) .
$$

We can now see that this mean-field approximation to the bus-route model is equivalent to a homogeneous zero-range process as discussed earlier in this section. Since $u(n)$ decays exponentially, with decay length $r=v / \lambda$, the condition for a strict phase transition is not met. It is reasonable to believe that the system behaves in a similar way to the system with a finite 'range' $r$ discussed in Section V.3. Since $r$ can be made arbitrarily large as $\lambda \longrightarrow 0$, on any finite system an apparent condensation will be seen. In the bus route problem this corresponds to the universally irritating situation of all the buses on the route arriving at once.

\section{Some further applications}

As mentioned earlier the zero-range process and related models have appeared several times in the modelling of nonequilibrium phenomena. Here we briefly discuss a few of these instances to illustrate the ubiquity of the basic model.

In [53] models of sandpile dynamics are considered. A zero range process is used to model the toppling of sand on a one-dimensional lattice; specifically the system is homogeneous and the occupation number of a site becomes the height of sand $(h)$ at that site. The hopping rates are set as $u(1)=1$ and $u(h)=\lambda$ for $h>1$, with the transition matrix a symmetric random walk, and the limit of large $\lambda$ considered. This limit means that a particle (grain of sand) keeps moving until it finds an unoccupied site, thus a hopping event may play the role of an avalanche. (Although in terms of sandpiles and self-organised criticality this model is rather trivial, it did serve to investigate the idea of a diverging diffusion constant.) Note that a slightly different $\lambda \rightarrow \infty$ limit (where the direction of the initial move of the particle is maintained until it finds an unoccupied site) was also considered but the product measure is still retained.

In a different context Barma and Ramaswamy [55] introduced the 'drop-push' model of activated flow involving transport through a series of traps. Each trap can only hold a finite number of particles. For the trap depth set equal to one this model is essentially the same as the sandpile model of [53] discussed above (i.e. it is a zero-range process with some infinite rates). In fact the version studied in [71] is precisely the limit of $u(n) \rightarrow \infty$ for $n>1$ of the totally asymmetric zero-range process described in Section III.1. A generalisation to inhomogeneous traps, and partially asymmetric hopping rates dependent on the occupancy of the trap was made in [72] and a steady state similar to $(11,17)$ demonstrated.

The zero-range process is also relevant in the context of $1+1$ dimensional interface growth by the step flow mechanism. The interface can be visualised as an ascending staircase of terraces. Adatoms land on the terraces and diffuse until they bind to the ascending step. If the ratio of deposition rates over diffusion rates tends to zero then the resulting dynamics is that a terrace shrinks by one unit (and the adjacent higher terrace grows by one unit) with a rate proportional to the size of the terrace. Thus the terrace lengths are equivalent to the site occupancies of an asymmetric zero-range process that was discussed in Section III.1. The equivalence of zero range processes to a general class of step flow models is discussed in [57].

Finally we note that the repton model of gelelectrophoresis [73] studied in the case of periodic boundary conditions by [52] is equivalent to an inhomogeneous zero-range process. In this case, the particles of the zero-range process represent the excess stored length of a polymer which diffuses along the tube of the polymer. The sites in the zero-range process represent the segments of the polymer tube and the inhomo- 
geneities in site hopping rates reflect the shape of the polymer tube.

\section{Conclusion}

In this work the aims were to give an overview of the area of phase transitions and ordering in onedimensional systems and also to analyse in some detail a particularly simple model, the zero-range process. In Section II several features were identified which could lead to the anomalous behaviour of ordering and phase transitions in equilibrium systems: long-range interactions; zero temperature; unbounded local variable. For nonequilibrium systems some concepts which may be important emerged: conserved order parameter; drive; forbidden microscopic transitions.

The simplicity of the zero-range process allowed us to analyse the steady state of the model in detail. First we derived the steady state for a general class of zerorange processes in Sections III and IV. We then analysed the condensation transitions that can occur. On an inhomogeneous system the condensation is very reminiscent of Bose-Einstein condensation. For it to occur requires certain conditions to hold on the distribution of hopping rates. In the homogeneous system the condensation corresponds to a spontaneous symmetry breaking, since an arbitrary site is selected to hold the condensate. The condition for it to occur is that the hopping rate dependence on the site occupancy decays sufficiently slowly. It was also shown that when the condition for condensation does not hold, one can still observe very sharp crossover behaviour and apparent condensation on a finite system

An interesting possibility that was explored was that of the existence of an effective energy function. We saw that any steady state of the form $(11,17)$ can be obtained from a process obeying detailed balance. However when the effective energy is inferred for cases where phase transition occurs (as was carried out for an explicit example in Section V.3) we find that it contains long-range interactions. Thus the condensation transition can be rationalised within the equilibrium framework.

Moreover in the zero-range process the existence of a drive or preferred direction, producing a conserved particle current, is not essential for the occurrence of a condensation transition. What does appear necessary, however, is the conservation of particles. The fixed number of particles implies implies the introduction of a fugacity $z$ through (32), which in turn controls the condensation transition. As we saw the fugacity gives the hopping rate out of a site (referred to as speed in Section V) which is a conserved quantity.

On the other hand, for other models the presence of a preferred direction and conserved current does seem crucial for the existence of phase transitions. For exam- ple, the asymmetric exclusion process defined in Section II.2 has non-trivial phase behaviour but the undriven version (symmetric exclusion) does not.

In summary, although a general theoretical framework for the description of phase transitions in one dimensional systems is not yet available, we hope that the issues and models discussed in the present paper serve to show that our understanding is developing.

\section{Acknowledgements}

Some of the work described here, particularly in Section II.2, has been the outcome of many enjoyable collaborations over the years and I thank all of my collaborators warmly. Special thanks are due to Owen O'Loan who kindly allowed me to borrow from parts of his $\mathrm{PhD}$ thesis in Sections V.2 and V.3 and with whom I have enjoyed many enlightening discussions. I also thank Isao Hiyane for pointing out the KDP model to me and Richard Blythe, Deepak Dhar and Joachim Krug for helpful comments on the manuscript.

\section{References}

[1] S. Katz, J. L. Lebowitz and H. Spohn Phys. Rev. B 28, 1655 (1983); J. Stat. Phys. 34, 497 (1984).

[2] B. Schmittmann and R. K. P. Zia (1995) Statistical Mechanics of Driven Diffusive Systems, edited by C. Domb and J.L. Lebowitz (Academic Press, U.K., 1995), Vol. 17; B. Schmittmann and R. K. P. Zia, Phys. Reports 301, 45 (1998).

[3] B. Bergesen, Z. Rácz and H-J Xu, Phys. Rev. E 52, 6031 (1995).

[4] F. J. Alexander and G. L. Eyink, Phys. Rev. E 57, R6229 (1998).

[5] J. Krug, Adv. Physics 46, 139 (1997).

[6] For an overview see Traffic and Granular Flow '97, edited by M. Schreckenberg and D. E. Wolf (Springer, Singapoure, 1998).

[7] K. Heckmann, Passive permeability of cell membranes, biomembranes (New York, Plenum Press, 1972) Vol. 3, p. 127 .

[8] T. Chou and D. Lohse, Phys. Rev. Lett. 82, 3552 (1999).

[9] C.T. MacDonald, J.H. Gibbs and A.C. Pipkin, Kinetics of biopolymerization on nucleic acid templates Biopolymers 6 1-25 (1968).

[10] F. Spitzer, Advances in Math. 5, 246 (1970).

[11] E. D. Andjel, Ann. Probability 10, 525 (1982).

[12] E. H. Lieb and D. C. Mattis, Mathematical Physics in One Dimension: Exactly Soluble Models of Interacting Particles (Academic Press, London, 1966), Chapter 1.

[13] L. D. Landau and E. M. Lifshitz, Statistical Physics I (Pergamon Press, New York, 1980). 
[14] M. E. Fisher, S.-k. Ma and B. G. Nickel, Phys. Rev. Lett. 29, 917 (1972).

[15] F. J. Dyson, Commun. Math. Phys. 12, 91 (1969).

[16] N. Goldenfeld, Lectures on Phase Transitions and the Renormalization Group (Addison Wesley, 1985).

[17] S. Karlin and H. M. Taylor, A First Course in Stochastic Processes (Academic, New York, 1975); F. R. Gantmacher, Matrix Theory (Chelsea, 1959) Vol. II, Chapter XIII.

[18] J.M.J van Leeuwen and H.J. Hilhorst, Physica A 107, 319 (1981).

[19] J. F. Nagle, Am. Journal of Physics 36, 1114 (1968).

[20] J. Krug, Phys. Rev. Lett. 67, 1882 (1991).

[21] B. Derrida, M. R. Evans, V. Hakim and V. Pasquier, J. Phys. A 26, 1493 (1993).

[22] G. Schütz and E. Domany, J. Stat. Phys. 72, 277 (1993).

[23] T. Sasamoto, J. Phys. A 32, 7109 (1999); condmat/9910270.

[24] R. A. Blythe, M. R. Evans, F. Colaiori and F. H. L. Essler (1999) cond-mat/9910242

[25] A. B. Kolomeisky, G. M. Schütz, E. B. Kolomeisky and J. P. Straley J. Phys. A 31, 6911 (1998).

[26] R. B. Stinchcombe and G. M. Schütz, Europhys. Lett. 29, 663 (1995).

[27] T. Sasamoto, S. Mori and M. Wadati, J. Phys. Soc. Japan 65, 2000 (1996).

[28] M. R. Evans, D. P. Foster, C. Godrèche, D. Mukamel, Phys. Rev. Lett. 74, 208 (1995).

[29] C. Godrèche, J-M. Luck, M. R. Evans, D. Mukamel, S. Sandow and E. R. Speer, J. Phys. A 28, 6039 (1995).

[30] For a review see S. A. Janowsky and J. L. Lebowitz, in Nonequilibrium Statistical Mechanics in One Dimension, edited by V. Privman (Cambridge University Press, U.K., 1997) Chapter 13, and references therein.

[31] B. Derrida, S.A. Janowsky, J.L. Lebowitz, E.R. Speer, J. Stat. Phys. 73, 813 (1993).

[32] Z. Toroczkai and R. K. P. Zia, Phys. Lett. A 217, 97 (1996).

[33] K. Mallick, J. Phys. A 29, 5375 (1996).

[34] B. Derrida and M. R. Evans, J. Phys. A 32, 4833 (1999).

[35] T. Sasamoto, (1999) cond-mat/9910483.

[36] B. Derrida, Phys. Reports. 301, 65 (1998).

[37] E. R. Speer, in Micro, Meso and Macroscopic Approaches in Physics, edited by C. Maes and A. Verbeure (Plenum, 1994).

[38] M. R. Evans, Y. Kafri, H. M. Koduvely and D. Mukamel, Phys. Rev. Lett. 80, 425 (1998); Phys. Rev. E 58, 2764.

[39] P. F. Arndt, T. Heinzel and V. Rittenberg, J. Phys. A 31, L45 (1998); cond-mat/9809123.

[40] R. Lahiri, M. Barma, S. Ramaswamy (1999) condmat/9907342.
[41] G. Korniss, B. Schmittmann and R. K. P. Zia, Europhys. Lett. 45, 431 (1999).

[42] L. Frachebourg, P. L. Krapivsky and E. Ben-Naim, Phys. Rev. E 54, 6186 (1996).

[43] H. Hinrichsen (2000). This volume.

[44] T. M. Liggett, Interacting Particle Systems (Springer, Berlin, 1985).

[45] J. Marro and R. Dickman, Nonequilibrium Phase Transitions in Lattice Models (Cambridge University Press, Cambridge, 1991).

[46] U. Alon, M.R. Evans, H. Hinrichsen and D. Mukamel (1996) Phys. Rev. Lett. 76, 2746 (1996).

[47] D. Helbing, D. Mukamel and G.M. Schütz, Phys. Rev. Lett. 82, 10 (1999).

[48] S. N. Majumdar, S. Krishnamurthy, M. Barma (1998) Phys. Rev. Lett. 81, 3691 (1998); cond-mat/9908403.

[49] A. Czirok, A-L. Barabasi, T. Vicsek, Phys. Rev. Lett. 81, 209 (1999).

[50] O. J. O'Loan and M. R. Evans, J. Phys. A 32, L99 (1999).

[51] H. Spohn (1991), Large Scale Dynamics of Interacting Particles, (Springer-Verlag, New York, 1991).

[52] J.M.J van Leeuwen and A. Kooiman, Physica, A 184, 79 (1992).

[53] J. M. Carlson, E. R. Grannan and G. H. Swindle, Phys. Rev. E, 47, 93 (1993).

[54] F. Ritort, Phys. Rev. Lett. 75, 1190 (1995).

[55] M. Barma and R. Ramaswamy in Non-linearity and Breakdown in Soft Condensed Matter, edited by K.K. Bardhan, B. K. Chakrabarti and A. Hansen (Springer, Berlin, 1993) p. 309.

[56] J. Krug and M. Schimschak, J. Phys I France, 5, 1065 (1995).

[57] J. Krug, in Nonequilibrium Statistical Mechanics in One Dimension, edited by V. Privman (Cambridge University Press, U.K., 1997), chapter 15.

[58] R. Kutner, K. W. Kehr, W. Renz and R. Przenioslo, J. Phys. A, 28, 923 (1995).

[59] M. R. Evans, J. Phys. A, 30, 5669 (1997).

[60] B. Derrida, J. Stat. Phys. 31, 433 (1983).

[61] I. Benjamini, P. A. Ferrari, C. Landim, Stoch. Proc. Appl. 61, 181 (1996).

[62] M. R. Evans, Europhys. Lett. 36, 13 (1996).

[63] J. Krug and P. A. Ferrari, J. Phys. A, 29, L465 (1996).

[64] For a clear exposition see e.g. K. Huang Statistical Mechanics, 2nd edition (Wiley, New York, 1987).

[65] J. Krug (2000). This volume.

[66] S. F. Burlatsky, G. Oshanin M. Morea and W. P. Reinhardt, Phys. Rev. E 54, 3165 (1996).

[67] L. H. Gwa and H. Spohn, Phys. Rev. A 46, 844 (1992).

[68] O. J. O'Loan PhD Thesis (University of Edinburgh, UK, 1999). 
[69] P. Bialas, Z. Burda and D. Johnston, Nucl. Phys. B 493, 505 (1997).

[70] O. J. O'Loan, M.R. Evans and M.E. Cates, Phys. Rev. E 58, 1404 (1998).

[71] G. M. Schütz, R. Ramaswamy and M. Barma, J. Phys. A 29, 837 (1996).
[72] G. Tripathy and M. Barma, Phys. Rev. E 58, 1991 (1998).

[73] M. Rubinstein, Phys. Rev. Lett. 59, 1496 (1987); T. A. J. Duke, Phys. Rev. Lett. 62, 2877 (1989). 